\title{
EXPLORING THE ROLE OF GLOBULAR CLUSTER SPECIFIC FREQUENCY ON THE NOVA RATES IN THREE VIRGO ELLIPTICAL GALAXIES
}

\author{
C. Curtin ${ }^{1,2}$, A. W. Shafter ${ }^{1}$, C. J. Pritchet ${ }^{3}$, J. D. Neill ${ }^{4}$, A. Kundu ${ }^{5,6}$, and T. J. Maccarone ${ }^{7}$ \\ ${ }^{1}$ Department of Astronomy and Mount Laguna Observatory, San Diego State University, San Diego, CA 92182, USA \\ ${ }^{2}$ Centre for Astrophysics and Supercomputing, Swinburne University of Technology, Hawthorn, VIC 3122, Australia \\ ${ }^{3}$ Department of Physics and Astronomy, University of Victoria, Victoria, BC V8W 2Y2, Canada \\ ${ }^{4}$ Space Radiation Laboratory 290-17, California Institute of Technology, Pasadena, CA 91125, USA \\ 5 Tata Institute of Fundamental Research, Mumbai 400005, India \\ ${ }^{6}$ Eureka Scientific Inc., 2452 Delmer Street, Suite 100, Oakland CA 94602, USA \\ ${ }^{7}$ Department of Physics, Texas Tech University, Box 41051, Lubbock TX 79409-1051, USA \\ Received 2015 May 15; accepted 2015 August 13; published 2015 September 17
}

\begin{abstract}
It has been proposed that a galaxy's nova rate might be enhanced by the production of nova progenitor binaries in the dense cores of its globular clusters (GCs). To explore this idea, relative nova rates in three Virgo elliptical galaxies, M87, M49, and M84, which have significantly different GC specific frequencies $\left(S_{N}\right)$ of 14, 3.6, and 1.6, respectively, were measured over the course of 4 epochs spanning a period of 14 months. To simplify the analysis, observations of the nearly equidistant galaxies were made on the same nights, with the same integration times, and through the same filter $(\mathrm{H} \alpha)$, so that the relative numbers of novae discovered would reflect the relative nova rates. At the conclusion of our survey we found a total of 27 novae associated with M87, 37 with M49, and 19 with M84. After correcting for survey completeness, we found annual nova rates of $154_{-19}^{+23}, 189_{-22}^{+26}$, and $95_{-14}^{+15}$, for M87, M49, and M84, respectively, corresponding to $K$-band luminosity-specific nova rates of $3.8 \pm 1.0,3.4 \pm 0.6$, and $3.0 \pm$ 0.6 novae per year per $10^{10} L_{K, \odot}$. The overall results of our study suggest that a galaxy's nova rate simply scales with its luminosity, and is insensitive to its GC specific frequency. Two novae, one in M87 and one in M84, were found to be spatially coincident with known GCs. After correcting for the mass fraction in GCs, we estimate that novae are likely enhanced relative to the field by at least an order of magnitude in the GC systems of luminous Virgo ellipticals.
\end{abstract}

Key words: galaxies: individual (M49, M84, M87) - galaxies: star clusters: general - novae, cataclysmic variables

\section{INTRODUCTION}

Classical novae are a subclass of cataclysmic variables in which a progenitor system increases in brightness by $\sim 10-20$ mag over a period of days to weeks and then returns to its preoutburst magnitude over a period of days to years. The progenitor system is a short period, semi-detached binary consisting of a white dwarf primary accreting material from a Roche lobe filling late-type companion. If the accretion rate is sufficiently low, the accreted material forms a thin shell of degenerate matter on the surface of the white dwarf. Once the temperature and pressure at the base of the accreted envelope become sufficiently high, a thermonuclear runaway ensues, resulting in a nova eruption. Novae can reach an absolute magnitude, $M_{V} \approx-10$, making them among the most luminous explosions in the universe, easily visible in galaxies as distant as the Virgo cluster (see Shafter 2008 for a review).

The nature of the progenitor system suggests that novae will be recurring events. Once a nova eruption occurs the material will begin to build up again and the process will repeat. Recurrence times can be as short as 1 year (e.g., see Darnley et al. 2014; Henze et al. 2014) all the way up to $10^{5}$ years, or perhaps longer. Despite the fact that all novae are believed to be recurrent, only novae with more than one recorded outburst are referred to as "recurrent novae." Over time, the orbital period of a nova progenitor binary decreases as a result of angular momentum losses due to magnetic stellar winds from the tidally locked donor star, or from gravitational wave radiation, or both. As the orbital period decreases, so does the average accretion rate onto the white dwarf primary, resulting in an increase in the recurrence time (Iben et al. 1992). In addition, in a given stellar population, the average mass of the primaries in nova systems are expected to decrease with increasing time elapsed since the zero-age main sequence population formed (Tutukov \& Yungelson 1995). A less massive primary needs to accrete more material to reach the necessary temperature near its surface to undergo a thermonuclear runaway (e.g., see Townsley \& Bildsten 2005). Such systems will undergo less frequent (and dimmer) nova outbursts compared to systems with higher mass primaries (Ritter et al. 1991; Livio 1992; Kolb 1995).

Accounting for the above factors, population synthesis models have predicted that $K$-band luminosity-specific nova rates, $\nu_{K}$, should be significantly higher in the younger populations found in spiral disks compared with older populations found in elliptical galaxies and in the bulges of spiral galaxies (e.g., Yungelson et al. 1997). Despite these predictions, many nova surveys have found that, within observational uncertainties (which can be large), $\nu_{K}$ appears to be relatively independent of Hubble type (e.g., Ciardullo et al. 1990b; Ferrarese et al. 2003; Williams \& Shafter 2004; Coelho et al. 2008; Güth et al. 2010; Franck et al. 2012), although some surveys have found evidence that $\nu_{K}$ varies between galaxies, though not necessarily as predicted by models (e.g., della Valle et al. 1994; Shara \& Zurek 2002; Madrid et al. 2007). In addition, nova surveys of M31, in which nearly 1000 novae have been observed (e.g., see Pietsch et al. 2007), have repeatedly found that the nova spatial distribution is more centrally concentrated than the background light, indicating a higher $\nu_{K}$ in the bulge than in the disk (e.g., Ciardullo et al. 1987; Capaccioli et al. 1989; Shafter \& Irby 
2001; Darnley et al. 2004, 2006). More recently Neill \& Shara (2004) have found a similar result for M81. In light of the apparent discrepancy between theory and observation found in their early M31 survey Ciardullo et al. (1987) suggested that a significant number of nova progenitor binaries may have formed in M31's globular cluster (GC) population and been subsequently injected into the bulge through 3-body encounters within clusters, or through tidal disruptions of entire clusters, or both, thereby enhancing the bulge nova rate.

It has long been known that the number of Galactic lowmass X-ray binaries per unit mass are enhanced in GC environments (Clark 1975; Katz 1975), and it has been suggested that many Galactic bulge X-ray sources could have had their origin in GCs (Grindlay 1988). A similar enhancement of X-ray sources has also been seen in M31's GC population (Crampton et al. 1984; Di Stefano et al. 2002). In recent years, it has been suggested that a significant population of close binaries with white dwarf accretors (e.g., nova progenitors) could also be formed in the dense cores of GCs (e.g., Pooley \& Hut 2006). Observations by the Hubble Space Telescope (HST) and Chandra had already begun to reveal these binaries in Galactic GCs (e.g., Edmonds et al. 2003; Heinke et al. 2003; Pooley et al. 2002; Knigge et al. 2002), although there is no compelling evidence that they are enhanced relative to the field.

Additional evidence for the idea that nova progenitors could be produced in GCs has come from the study of nova rates in Virgo elliptical galaxies. Shara \& Zurek (2002) reported that the nova rate in the giant elliptical galaxy M87 could be as high as $\sim 300 \mathrm{yr}^{-1}$, while for the slightly more luminous elliptical, M49, Ferrarese et al. (2003) found a rate of $R \approx 100$. It is interesting that, like the ratio of observed nova rates, M87 has a specific frequency of GCs about three times that of M49 (Brodie \& Strader 2006). Although, these results are consistent with the possibility that the large GC population of M87 enhances its nova rate, absolute nova rates extrapolated from observed nova rates are largely uncertain given the small spatial coverage of these HST surveys, and comparisons of these rates between surveys can be unreliable.

In 2011 we began a definitive test of the putative relationship between a galaxy's GC specific frequency, $S_{N}$, and its luminosity-specific nova rate, $\nu_{K}$. Specifically, we searched for novae in three essentially equidistant Virgo elliptical galaxies having different GC specific frequencies. The galaxies were observed under nearly identical conditions using the same telescope, observing cadence, filters, and exposure times. With this approach we were able to simply compare the relative numbers of novae discovered in each of the three galaxies directly to estimate relative nova rates, while avoiding the large uncertainties that result when attempting to measure absolute nova rates for different galaxies in different surveys. Preliminary results of our program were discussed in Shafter et al. (2013) and Curtin et al. (2014). Here, we present the final results of the first phase of our ongoing Virgo galaxy nova study.

\section{OBSERVATIONS}

We chose three Virgo ellipticals for our study: M87 (NGC 4486), M49 (NGC 4472), and M84 (NGC 4374). As members of the Virgo cluster, these three galaxies are approximately equidistant. They also have similar luminosities, but they have widely varying GC specific frequencies, $S_{N}$ (see Table 1). We
Table 1

Galaxy Properties

\begin{tabular}{lccccc}
\hline \hline Galaxy & $S_{N}$ & $\begin{array}{c}(m-M) \\
(\mathrm{mag})\end{array}$ & $\begin{array}{c}B_{T, 0} \\
(\mathrm{mag})\end{array}$ & $\begin{array}{c}(B-V)_{0} \\
(\mathrm{mag})\end{array}$ & $\begin{array}{c}(V-K)_{0} \\
(\mathrm{mag})\end{array}$ \\
\hline M87 & 14 & 31.03 & 9.49 & 0.96 & 3.21 \\
M49 & 3.6 & 31.06 & 9.28 & 0.96 & 3.34 \\
M84 & 1.6 & 31.32 & 9.99 & 0.87 & 3.28 \\
\hline
\end{tabular}

Note. $S_{N}$ are from Brodie \& Strader (2006), $(m-M)$ are from Tonry et al. (2001), $B_{T, 0}$ and $(B-V)_{0}$ are from de Vaucouleurs et al. (1991), and $(V-K)_{0}$ are from Frogel et al. (1978).

Table 2

Observations

\begin{tabular}{|c|c|c|c|c|c|}
\hline Epoch & Date & Galaxy & Exp & Seeing & Weather \\
\hline \multirow[t]{3}{*}{1} & 2011 Feb 02 & M49 & $12 \times 5 \mathrm{~m}$ & $0 " .99$ & phot \\
\hline & 2011 Feb 02 & M84 & $12 \times 5 \mathrm{~m}$ & $0 " .82$ & phot \\
\hline & 2011 Jan 30 & M87 & $12 \times 5 \mathrm{~m}$ & $0 " .87$ & phot \\
\hline \multirow[t]{3}{*}{2} & 2011 Mar 30 & M49 & $12 \times 5 \mathrm{~m}$ & $0 " .82$ & phot \\
\hline & 2011 Apr 02 & M84 & $12 \times 5 \mathrm{~m}$ & $0 " .89$ & phot \\
\hline & 2011 Mar 29 & M87 & $16 \times 5 \mathrm{~m}$ & $0 " .95$ & p cldy \\
\hline \multirow[t]{3}{*}{3} & 2012 Feb 26 & M49 & $11 \times 5 \mathrm{~m}$ & $1 " 19$ & phot \\
\hline & 2012 Feb 27 & M84 & $12 \times 5 \mathrm{~m}$ & $0 " 72$ & p cldy \\
\hline & 2012 Feb 26 & M87 & $12 \times 5 \mathrm{~m}$ & $0 " .96$ & phot \\
\hline \multirow[t]{3}{*}{4} & 2012 Mar 21 & M49 & $15 \times 5 \mathrm{~m}$ & $0 " .72$ & p cldy \\
\hline & 2012 Mar 22 & M84 & $12 \times 5 \mathrm{~m}$ & $0 " .66$ & p cldy \\
\hline & $2012 \operatorname{Mar} 21$ & M87 & $13 \times 5 \mathrm{~m}$ & $0 " .81$ & p cldy \\
\hline
\end{tabular}

observed all three galaxies over four epochs from 2011 February to 2012 March using the Canada-France-Hawaii $3.6 \mathrm{~m}$ telescope (CFHT). All observations were made using the MegaCam which consists of a mosaic of $362048 \times 4612$ pixel CCDs covering a full field of view of $\sim 1 \mathrm{deg}^{2}$ at a resolution of 0". 187/pixel (Boulade et al. 2003). Exposure times were kept nearly constant between galaxies, varying only slightly due to weather in a small number of cases to ensure that the derived relative nova rates would be directly comparable. The observations are summarized in Table 2 .

We chose to image all three galaxies through a narrow-band $\mathrm{H} \alpha$ filter redshifted to the mean velocity of the Virgo cluster $\left(\lambda_{c}=6584 \AA\right.$, FWHM $\left.=76 \AA\left[\sim 3500 \mathrm{~km} \mathrm{~s}^{-1}\right]\right)$. For nova surveys imaging in $\mathrm{H} \alpha$ has two principal advantages over broad-band imaging. Novae exhibit strong $\mathrm{H} \alpha$ emission lines shortly after outburst that fade slowly relative to the continuum, typically requiring months to decline by more than 2 mag. The slow decay rate in $\mathrm{H} \alpha$ means that we can detect novae over a longer time interval, requiring less frequent temporal sampling. Another advantage of $\mathrm{H} \alpha$ over broad-band observations, is that $\mathrm{H} \alpha$ images provide a greater contrast between novae and the bright galaxy background, resulting in increased survey completeness, particularly near the bright nuclei of galaxies (e.g., Ciardullo et al. 1987).

Based on the luminosity function of novae in both M31 and the Galaxy, we estimate that approximately $50 \%$ of the Virgo novae should reach a peak absolute magnitude, $M_{\text {max }, \mathrm{H} \alpha}=-7.5 .^{8}$ Assuming a distance modulus to the Virgo cluster of $\mu_{0} \simeq 31.0$ (see Table 1 ), we require observations that can reach $m_{\mathrm{H} \alpha} \simeq 23.5$. Numerical simulations revealed that

\footnotetext{
8 The $\mathrm{H} \alpha$ magnitude is defined on the $\mathrm{AB}$ system where $m_{\mathrm{H} \alpha}=0$ for $f_{\lambda}=2.53 \times 10^{-9} \mathrm{erg} \mathrm{cm}^{-2} \mathrm{~s}^{-1} \AA^{-1}$. Through a $76 \AA$ wide filter, this corresponds to a zero point flux of $\sim 1.9 \times 10^{-7} \mathrm{erg} \mathrm{cm}^{-2} \mathrm{~s}^{-1}$.
} 
with a mean seeing of 0 ". 8 typical for the CFHT we could detect a $m=23.5$ nova during gray time with a signal-to-noise ratio, $\mathrm{S} / \mathrm{N}, \sim 12$ at $60^{\prime \prime}$ from the center of its host galaxy and $\mathrm{S} / \mathrm{N} \sim 6$ at $30^{\prime \prime}$ from center in a one-hour exposure. To avoid saturation of the nucleus, and to enable the removal of cosmic ray hits, our $1 \mathrm{hr}$ exposures were divided into 12 five-minute integrations.

Our complete survey was spread over four epochs of observations spanning two years. The first two epochs were separated by two months, which, given the persistence of the nova $\mathrm{H} \alpha$ emission, provides reasonable temporal coverage, with minimal overlap of detected novae. The third and fourth epochs were scheduled in the following year to ensure that we could discriminate between slowly evolving novae and other types of variable sources.

Given an estimate for the absolute nova rate in M49 (100 $\mathrm{yr}^{-1}$, Ferrarese et al. 2003), we conducted a simulation to estimate the number of novae we could expect to observe in each epoch. In the simulation, we consider de Vaucouleurs (1948) model for the brightness profile of the galaxy and then distribute artificial novae with a density that follows the background light. The peak brightnesses and fade rates for the artificial novae are based on $\mathrm{H} \alpha$ light curves of 14 novae observed in M31 (Shafter \& Irby 2001) and M81 (Neill \& Shara 2004), adjusted to the distance of the Virgo cluster. Given the temporal sampling, exposure times (see Table 2) and an estimate of the nova rate we compute the number of novae expected to be observed above a given $\mathrm{S} / \mathrm{N}$. Under the null hypothesis that the nova rate is independent of GC specific frequency, and scales directly with $K$-band luminosity, we would expect to observe 22, 32 and 15 novae in M87, M49, and $\mathrm{M} 84$, respectively with a $\mathrm{S} / \mathrm{N} \geqslant 10$. On the other hand, in the extreme (and likely unrealistic) case that all nova progenitors are formed in GCs, the number of novae expected scales directly with the GC specific frequency and the predicted rates were 86,32 and 7 novae for the three galaxies, respectively.

\section{DATA REDUCTION AND ANALYSIS}

In each observation, we positioned the target galaxy on the top of CCD chip 22 near the center of the MegaCam array. More than $99 \%$ of the total light from each galaxy falls on the same six central chips surrounding the nucleus (chips 12, 13, $14,21,22,23)$, and these chips were the focus of our analysis. The images were not significantly dithered, and so chip gaps of $\sim 13^{\prime \prime}$ persist in the final images for each epoch. The area obscured by chip gaps is $\sim 3 \%$ of the total survey area. However, due to the positioning of the target galaxies, no chip gaps are encountered within a radius of $120^{\prime \prime}$ of the nuclei. Using the same photometry used to plot the cumulative background light in Figure 2, we estimate the light enclosed within this radius to be greater than $60 \%$ of the total light for all targets, leaving considerably less than $1.2 \%$ of the light (and likely the novae) obscured by chip gaps. This loss is negligible compared to other uncertainties inherent in nova rate calculations, and we have not made any formal correction for it in the analysis.

\footnotetext{
9 IRAF is distributed by the National Optical Astronomy Observatory, which is operated by Association of Universities for Research in Astronomy, Inc. under cooperative agreement with the National Science Foundation.
}

The MegaCam data come pre-processed with bad pixel masking, bias subtraction, and flat fielding being performed prior to the data release. Once the pre-processed data were in hand, individual 5 minute exposures for each of the six central chips were spatially registered using the $\operatorname{IRAF}^{9}$ routine WREGISTER. The individual CCD images were then median stacked using the tasks IMALIGN and IMCOMBINE to produce master images for each chip in each epoch, typically representing 60 minute of total exposure time (see Table 2). Finally, the WCS for each master image was recalibrated to match between epochs using the IRAF task CCMAP in conjunction with precise coordinates for several reference stars on each image obtained from the US Naval Observatory (USNO) B1 catalog (Monet et al. 2003).

\subsection{Nova Detection}

To identify nova candidates the master images were spatially aligned, point-spread function (PSF) matched and differenced using the ISIS package (Alard \& Lupton 1998). In the resulting images objects of variable brightness are conspicuous, allowing us to detect them by eye. Variables qualified as nova candidates if they had an approximately Gaussian PSF, and if they were visible in only one or two consecutive epochs, but did not appear on both sides of the 10 month gap in observations.

Within the inner $\sim 30^{\prime \prime}$ of each galaxy center the differencing became less effective due to the increasingly bright background light. In an attempt to detect nova candidates in the inner regions we applied the IRAF task MEDIAN, which produced a smoothed approximation of the background light profile. Stellar sources are not completely removed in the median smoothing process, but they are greatly reduced in amplitude. The resulting median image was then subtracted from the original to produce a background-subtracted image of the central region where stellar sources are easier to identify. The background-subtracted images from separate epochs were then compared to identify nova candidates. No new nova candidates were identified using this method, however we were able to confirm pre-existing candidates or in a couple of cases eliminate candidates due to non-stellar PSFs first identified in the background subtracted images. All told, we have discovered a total of 27, 37, and 19 novae in the fields of M87, M49, and M84.

\subsection{Photometry}

To calibrate the nova $\mathrm{H} \alpha$ magnitudes, we began by performing aperture photometry using the IRAF task $\mathrm{PHOT}$ to determine instrumental magnitudes for the nova candidates and several nearby reference stars from the USNO B1 catalog, which were employed as secondary standards. For nova candidates close to the nucleus we needed to account for the steepness of the background light profile before we could perform accurate aperture photometry. To ensure that we were able to accurately measure the nova brightness, we preprocessed subimages centered on the nova using the task IMSURFIT, which excludes a small circular region surrounding the nova and then calculates and subtracts a fit of the background light.

Once instrumental magnitudes were in hand, standard AB magnitudes for the novae (which correspond to monochromatic fluxes averaged over the $\mathrm{H} \alpha$ filter bandpass) were estimated through differential photometry with respect to the known $R$ - 
Table 3

M87 Novae

\begin{tabular}{|c|c|c|c|c|c|}
\hline $\begin{array}{l}\text { Nova } \\
\text { N4486 }\end{array}$ & MJD & $\begin{array}{c}\alpha \\
(\mathrm{J} 2000)\end{array}$ & $\begin{array}{c}\delta \\
(\mathrm{J} 2000)\end{array}$ & $\begin{array}{c}m_{\mathrm{H} \alpha} \\
(\mathrm{mag})\end{array}$ & $\begin{array}{l}a \\
\left({ }^{\prime}\right)\end{array}$ \\
\hline 2011-01a & 55590.9 & $12: 30: 26.4$ & $12: 24: 50$ & 22.8 & 6.97 \\
\hline 2011-01b & $\ldots$ & $12: 30: 36.9$ & $12: 24: 05$ & 22.2 & 3.76 \\
\hline 2011-01c & $\cdots$ & $12: 30: 43.5$ & $12: 22: 59$ & 22.0 & 1.77 \\
\hline 2011-01d & $\ldots$ & $12: 30: 44.3$ & $12: 24: 53$ & 22.4 & 1.91 \\
\hline 2011-01e & $\ldots$ & $12: 30: 49.1$ & $12: 23: 08$ & 21.4 & 0.35 \\
\hline 2011-01f & $\ldots$ & $12: 30: 49.7$ & $12: 22: 04$ & 22.3 & 1.44 \\
\hline $2011-01 \mathrm{~g}$ & $\ldots$ & $12: 31: 26.1$ & $12: 21: 06$ & 22.3 & 11.12 \\
\hline 2011-03a & 55646.0 & $12: 30: 23.8$ & $12: 17: 22$ & 21.2 & 11.01 \\
\hline $2011-03 b$ & $\cdots$ & $12: 30: 37.0$ & $12: 21: 47$ & 21.7 & 4.49 \\
\hline $2011-03 c$ & $\ldots$ & $12: 30: 46.0$ & $12: 19: 35$ & 22.4 & 4.38 \\
\hline 2011-03d & $\ldots$ & $12: 30: 52.2$ & $12: 22: 13$ & 23.0 & 1.42 \\
\hline $2011-03 \mathrm{e}$ & $\ldots$ & $12: 30: 52.3$ & $12: 21: 36$ & 22.3 & 2.00 \\
\hline 2011-03f & $\ldots$ & $12: 30: 56.3$ & $12: 13: 19$ & 22.7 & 10.46 \\
\hline $2012-02 a$ & 55983.0 & $12: 30: 44.1$ & $12: 24: 01$ & 23.2 & 1.50 \\
\hline 2012-02b & $\cdots$ & $12: 30: 44.4$ & $12: 21: 18$ & 22.1 & 2.89 \\
\hline $2012-02 c$ & $\ldots$ & $12: 30: 44.5$ & $12: 23: 03$ & 22.8 & 1.46 \\
\hline $2012-02 d$ & $\ldots$ & $12: 30: 47.3$ & $12: 24: 45$ & 22.0 & 1.39 \\
\hline $2012-02 \mathrm{e}$ & $\ldots$ & $12: 30: 51.3$ & $12: 23: 47$ & 21.5 & 0.59 \\
\hline 2012-02f & $\ldots$ & $12: 30: 55.0$ & $12: 20: 55$ & 23.2 & 2.90 \\
\hline $2012-02 \mathrm{~g}$ & $\ldots$ & $12: 30: 56.2$ & $12: 31: 15$ & 22.9 & 8.77 \\
\hline $2012-03 a$ & 56006.9 & $12: 30: 14.1$ & $12: 35: 50$ & 22.8 & 15.33 \\
\hline $2012-03 b$ & $\ldots$ & $12: 30: 28.9$ & 12:38:09 & 22.4 & 15.53 \\
\hline $2012-03 c$ & $\ldots$ & $12: 30: 45.2$ & $12: 23: 54$ & 21.9 & 1.18 \\
\hline $2012-03 d^{a}$ & $\ldots$ & $12: 30: 51.2$ & $12: 21: 59$ & 22.5 & 1.56 \\
\hline $2012-03 e$ & $\cdots$ & $12: 30: 52.7$ & $12: 25: 06$ & 21.0 & 2.03 \\
\hline 2012-03f & $\ldots$ & $12: 30: 58.5$ & $12: 22: 57$ & 22.9 & 2.60 \\
\hline 2012-03g & $\ldots$ & $12: 31: 26.0$ & $12: 21: 46$ & 22.7 & 11.08 \\
\hline
\end{tabular}

Notes. The units for R.A. are hours, minutes, seconds. The units for decl. are degrees, arcminutes, arcseconds. Apparent magnitudes are approximate only. The parameter $a$ is the isophotal semimajor axis of the nova.

${ }^{\mathrm{a}}$ This nova was detected coincident with GC M87:[PJB2009] 1667.

band magnitudes of the reference stars. Conversion to a true $\mathrm{H} \alpha$ magnitude that would reflect the total nova emission at $\mathrm{H} \alpha$ would require a knowledge of the width of the nova $\mathrm{H} \alpha$ emission relative to the filter bandpass. Since the former value varies from nova to nova depending on the expansion velocity of the nova shell, an accurate measure of a nova's $\mathrm{H} \alpha$ flux through filter photometry is not possible. For consistency with our earlier studies (e.g., see Ciardullo et al. 1990a; Shafter et al. 2000), we assume a 100\% filling fraction in the present analysis. In this case, the adopted nova $\mathrm{H} \alpha$ magnitudes are simply equivalent to standard $\mathrm{AB}$ magnitudes, with $m_{\mathrm{H} \alpha}=0$ corresponding to $f_{\lambda}=2.53 \times 10^{-9} \mathrm{erg} \mathrm{cm}^{-2} \mathrm{~s}^{-1} \AA^{-1}$.

Equatorial coordinates, the $\mathrm{H} \alpha$ magnitude, and the isophotal semimajor axis, $a$, are given in Tables 3-5 for the three galaxies, respectively.

\subsection{Spatial Distributions, Completeness, and Galaxy Membership}

The spatial positions of the novae detected in each of the three galaxies are shown in Figure 1. Given that the Virgo galaxies are members of a rich cluster, it is possible that some fraction of the novae, especially those seen at a large distance from their putative host galaxy, may in fact be associated with nearby dwarf galaxies, or possibly with intracluster novae (e.g.,
Table 4

M49 Novae

\begin{tabular}{|c|c|c|c|c|c|}
\hline $\begin{array}{l}\text { Nova } \\
\text { N4472 }\end{array}$ & MJD & $\begin{array}{c}\alpha \\
(\mathrm{J} 2000.0)\end{array}$ & $\begin{array}{c}\delta \\
(\mathrm{J} 2000.0)\end{array}$ & $\begin{array}{c}m_{\mathrm{H} \alpha} \\
(\mathrm{mag})\end{array}$ & $\begin{array}{l}a \\
(')\end{array}$ \\
\hline $2011-02 a$ & 55593.9 & $12: 29: 42.5$ & $07: 57: 58$ & 22.2 & 2.63 \\
\hline $2011-02 b$ & $\ldots$ & $12: 29: 43.4$ & $07: 59: 17$ & 21.9 & 1.37 \\
\hline $2011-02 c$ & $\ldots$ & $12: 29: 46.2$ & 08:01:25 & 22.7 & 1.44 \\
\hline $2011-02 d$ & $\ldots$ & $12: 29: 47.7$ & 08:01:44 & 22.9 & 1.84 \\
\hline $2011-02 \mathrm{e}$ & $\ldots$ & $12: 30: 08.4$ & $07: 58: 17$ & 21.4 & 6.50 \\
\hline $2011-03 a$ & 55650.0 & $12: 29: 30.7$ & $08: 06: 24$ & 21.5 & 7.53 \\
\hline $2011-03 b$ & $\ldots$ & $12: 29: 34.7$ & $07: 57: 30$ & 22.3 & 4.87 \\
\hline $2011-03 c$ & $\ldots$ & $12: 29: 37.7$ & 08:00:15 & 21.2 & 2.61 \\
\hline $2011-03 d$ & $\ldots$ & $12: 29: 37.9$ & $07: 59.04$ & 22.3 & 2.91 \\
\hline $2011-03 \mathrm{e}$ & $\ldots$ & $12: 29: 42.1$ & 08:02:54 & 21.4 & 3.09 \\
\hline 2011-03f & $\ldots$ & $12: 29: 46.4$ & 08:04:41 & 23.3 & 4.87 \\
\hline $2011-03 \mathrm{~g}$ & $\ldots$ & $12: 29: 47.2$ & $07: 59: 30$ & 21.7 & 0.55 \\
\hline $2011-03 \mathrm{~h}$ & $\ldots$ & $12: 29: 48.1$ & $07: 59: 32$ & 21.2 & 0.62 \\
\hline $2011-03 i$ & $\ldots$ & $12: 29: 50.3$ & 07:59:10 & 22.9 & 1.29 \\
\hline 2011-03j & $\ldots$ & $12: 29: 52.5$ & $07: 58: 58$ & 23.2 & 1.89 \\
\hline $2011-03 k$ & $\ldots$ & $12: 29: 52.7$ & 08:01:46 & 23.2 & 2.70 \\
\hline 2011-031 & $\ldots$ & $12: 29: 53.2$ & 08:00:09 & 22.6 & 1.93 \\
\hline $2012-02 a$ & 55983.1 & $12: 29: 07.3$ & $08: 13: 10$ & 22.4 & 16.57 \\
\hline $2012-02 b$ & $\ldots$ & $12: 29: 36.6$ & 07:58:50 & 21.9 & 3.46 \\
\hline $2012-02 c$ & $\ldots$ & $12: 29: 44.5$ & $07: 59: 21$ & 22.7 & 1.01 \\
\hline $2012-02 d$ & $\ldots$ & $12: 29: 47.4$ & 07:58:40 & 22.2 & 1.42 \\
\hline $2012-02 \mathrm{e}$ & $\ldots$ & $12: 29: 51.1$ & 08:00:04 & 22.0 & 1.33 \\
\hline $2012-02 f$ & $\ldots$ & $12: 29: 56.9$ & $07: 54: 48$ & 22.5 & 5.81 \\
\hline $2012-02 \mathrm{~g}$ & $\ldots$ & $12: 30: 12.7$ & $07: 55: 59$ & 22.7 & 8.26 \\
\hline $2012-03 a$ & 56007.0 & $12: 29: 18.4$ & 08:09:34 & 23.5 & 11.97 \\
\hline $2012-03 b$ & $\ldots$ & $12: 29: 44.4$ & $08: 02: 36$ & 22.6 & 2.66 \\
\hline $2012-03 c$ & $\ldots$ & $12: 29: 44.7$ & 08:03:02 & 22.5 & 3.10 \\
\hline $2012-03 d$ & $\ldots$ & $12: 29: 46.0$ & 08:00:45 & 22.6 & 0.74 \\
\hline $2012-03 \mathrm{e}$ & $\ldots$ & $12: 29: 47.9$ & 08:01:39 & 22.0 & 1.78 \\
\hline 2012-03f & $\ldots$ & $12: 29: 49.4$ & 08:02:08 & 21.5 & 2.42 \\
\hline $2012-03 \mathrm{~g}$ & $\ldots$ & $12: 29: 50.9$ & $07: 58: 38$ & 22.6 & 1.76 \\
\hline $2012-03 \mathrm{~h}$ & $\ldots$ & $12: 29: 53.6$ & 08:00:51 & 23.5 & 2.28 \\
\hline $2012-03 i$ & $\ldots$ & $12: 29: 54.0$ & 08:00:23 & 22.7 & 2.20 \\
\hline $2012-03 j$ & $\ldots$ & $12: 29: 57.0$ & 07:58:08 & 21.8 & 3.36 \\
\hline $2012-03 k$ & $\ldots$ & $12: 29: 57.5$ & $07: 56: 46$ & 21.3 & 4.30 \\
\hline $2012-031$ & $\ldots$ & $12: 29: 58.2$ & $07: 58: 23$ & 21.6 & 3.58 \\
\hline $2012-03 \mathrm{~m}$ & $\ldots$ & 12:30:06.1 & 08:04:02 & 21.9 & 8.81 \\
\hline
\end{tabular}

Note. See notes for Table 3.

see Neill et al. 2005; Shara 2006). To pursue this possibility further, and to explore how the nova populations are distributed in each of their host galaxies, in Figure 2 we have compared the radial cumulative distribution of the novae with the cumulative distribution of the galaxy's background light using the $g$-band photometry of Cohen (1986) for M87 and M49, and the $B$-band photometry of Michard (1985) for M84. The different bandpass used for the M84 photometry should not affect the analysis significantly given that color gradients in the Virgo ellipticals are not appreciable (e.g., see Cohen 1986). In each case the inner value of the isophotal semimajor axis, $a_{\text {in }}$, was set to be the minimum value at which novae could be detected. Artificial star tests discussed below in Section 4.2 show that our nova samples are complete to the limiting magnitude beyond $a_{\text {in }}=1^{\prime}$ for M87, $a_{\text {in }}=1^{\prime} .4$ for M 49 , and $a_{\text {in }}=0.5$ for M84. Since our surveys were always incomplete within $0.5^{\prime}$ of the center of each galaxy, we cannot address the possibility that the nova density is enhanced near the nuclei of the galaxies, as was suggested by Madrid et al. (2007) in their HST study of 
Table 5

M84 Novae

\begin{tabular}{lccccc}
\hline \hline $\begin{array}{l}\text { Nova } \\
\text { N4374 }\end{array}$ & MJD & $\begin{array}{c}\alpha \\
(\mathrm{J} 2000.0)\end{array}$ & $\begin{array}{c}\delta \\
(\mathrm{J} 2000.0)\end{array}$ & $\begin{array}{c}m_{\mathrm{H} \alpha} \\
(\mathrm{mag})\end{array}$ & $\begin{array}{c}a \\
\left({ }^{\prime}\right)\end{array}$ \\
\hline $2011-02 \mathrm{a}$ & 55594.9 & $12: 24: 40.8$ & $12: 53: 12$ & 23.4 & 5.65 \\
$2011-02 \mathrm{~b}$ & $\ldots$ & $12: 24: 54.3$ & $12: 52: 38$ & 23.4 & 2.43 \\
$2011-02 \mathrm{c}$ & $\ldots$ & $12: 25: 07.1$ & $12: 53: 28$ & 21.8 & 0.92 \\
$2011-04 \mathrm{a}$ & 55652.8 & $12: 24: 47.6$ & $12: 52: 07$ & 22.9 & 4.19 \\
$2011-04 \mathrm{~b}^{\mathrm{a}}$ & $\ldots$ & $12: 25: 02.2$ & $12: 53: 43$ & 22.9 & 0.63 \\
$2011-04 \mathrm{c}$ & $\ldots$ & $12: 25: 03.5$ & $12: 52: 18$ & 23.0 & 0.98 \\
$2011-04 \mathrm{~d}$ & $\ldots$ & $12: 25: 15.1$ & $12: 52: 28$ & 21.8 & 2.88 \\
$2012-02 \mathrm{a}$ & 55984.1 & $12: 25: 00.3$ & $12: 52: 33$ & 23.7 & 1.14 \\
$2012-02 \mathrm{~b}$ & $\ldots$ & $12: 25: 00.7$ & $12: 53: 49$ & 23.0 & 0.95 \\
$2012-02 \mathrm{c}$ & $\ldots$ & $12: 25: 01.0$ & $12: 55: 15$ & 23.2 & 2.21 \\
$2012-02 \mathrm{~d}$ & $\ldots$ & $12: 25: 01.8$ & $12: 53: 48$ & 22.8 & 0.75 \\
$2012-02 \mathrm{e}$ & $\ldots$ & $12: 25: 02.6$ & $12: 53: 19$ & 22.3 & 0.29 \\
$2012-02 \mathrm{f}$ & $\ldots$ & $12: 25: 02.6$ & $12: 53: 32$ & 22.9 & 0.42 \\
$2012-02 \mathrm{~g}$ & $\ldots$ & $12: 25: 19.6$ & $12: 57: 48$ & 22.7 & 6.37 \\
$2012-03 \mathrm{a}$ & 56007.9 & $12: 24: 47.0$ & $12: 58: 38$ & 23.0 & 6.87 \\
$2012-03 \mathrm{~b}$ & $\ldots$ & $12: 25: 04.2$ & $12: 52: 53$ & 21.9 & 0.37 \\
$2012-03 \mathrm{c}$ & $\ldots$ & $12: 25: 05.1$ & $12: 53: 22$ & 22.5 & 0.42 \\
$2012-03 \mathrm{~d}$ & $\ldots$ & $12: 25: 06.2$ & $12: 57: 57$ & 22.0 & 5.05 \\
$2012-03 \mathrm{e}$ & $\ldots$ & $12: 25: 07.8$ & $12: 53: 09$ & 23.3 & 1.03 \\
& & & & & \\
\end{tabular}

Notes. See notes for Table 3 .

${ }^{\mathrm{a}}$ This nova was detected coincident with a GC, NGC 4374:[CLW2011] 033.

novae in the inner regions of M87, and by Ferrarese et al. (2003) for M49.

If the number density of nova progenitor binaries is simply proportional to the number density of stars, we would expect the cumulative nova distribution as a function of isophotal semimajor axis to closely trace that of the galactic background light. Based on an examination of the cumulative distribution plots, including their Kolmogorov-Smirnoff (KS) statistics, it appears that the density of novae follows the background light reasonably well. In the case of M87 there seems to possibly be two separate populations present. The M87 distribution is smooth from the central region to $a \sim 10^{\prime}$, then there is a gap in the distribution followed by what appears to be an increase in the nova density at $a \gtrsim 10^{\prime}$. A visual inspection of the M87 field reveals that within the region of this outer population several small neighboring galaxies are being included in the survey, such as NGC 4478 which by itself is $\sim 10 \%$ the luminosity of M87. One of the novae in the outer population lies within $\sim 2$ ". 7 of NGC 4478 . There is also evidence for an extended halo surrounding M87 (e.g., see Kormendy et al. 2009). Thus is it possible that these outer novae are not directly associated with M87.

Given the relatively poor fit of the nova distribution to the cumulative light in the case of M87 we have considered a truncated sample of novae with $a<10^{\prime}$ consisting of 21 novae for which there is a high probability of direct association with the galaxy. The revised cumulative distribution is shown in Figure 3. After removing six novae with $a>10^{\prime}$ and two novae with $a<1^{\prime}$ and rescaling the remaining novae to the background light accordingly, the KS statistic between the background light and the nova distribution for M87 improves markedly ( $\mathrm{KS}=81 \%)$.
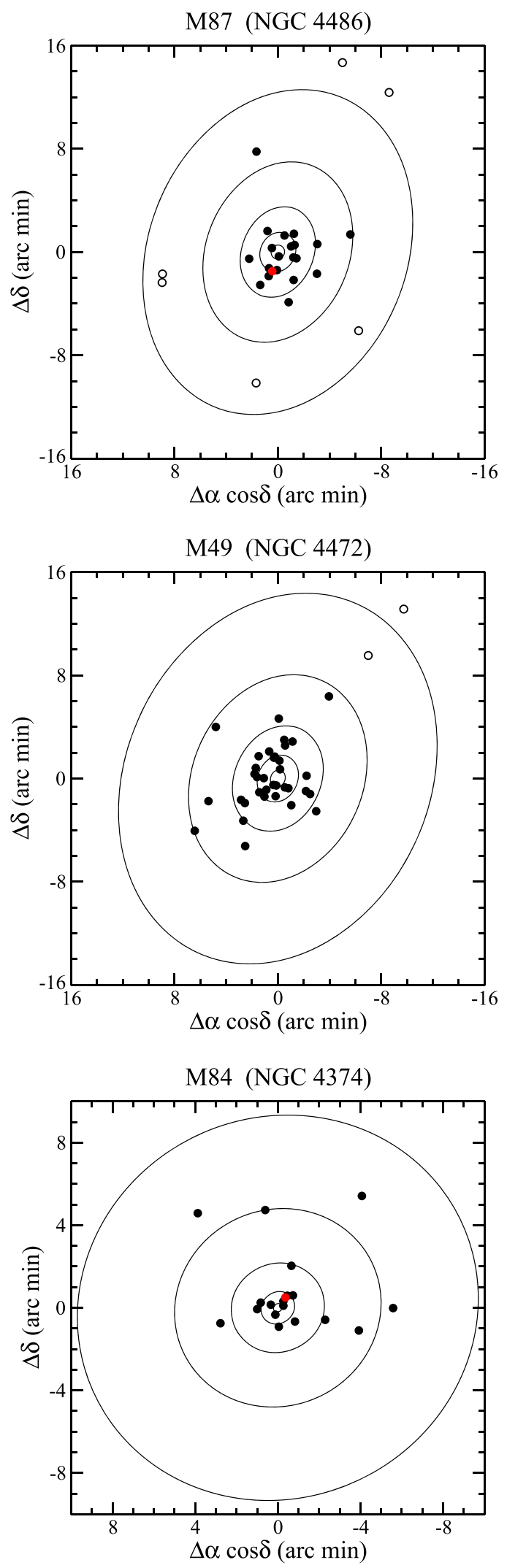

Figure 1. Spatial positions of the novae detected in each of our surveyed galaxies, plotted over equally spaced isophotes from $\mu=20$ to $\mu=28 \mathrm{mag}$ $\operatorname{arcsec}^{-2}$. Open circles represent novae that are more than $10^{\prime}$ from the nucleus, which have been excluded from the second analysis of M87. Filled red circles mark the locations of GC novae. 

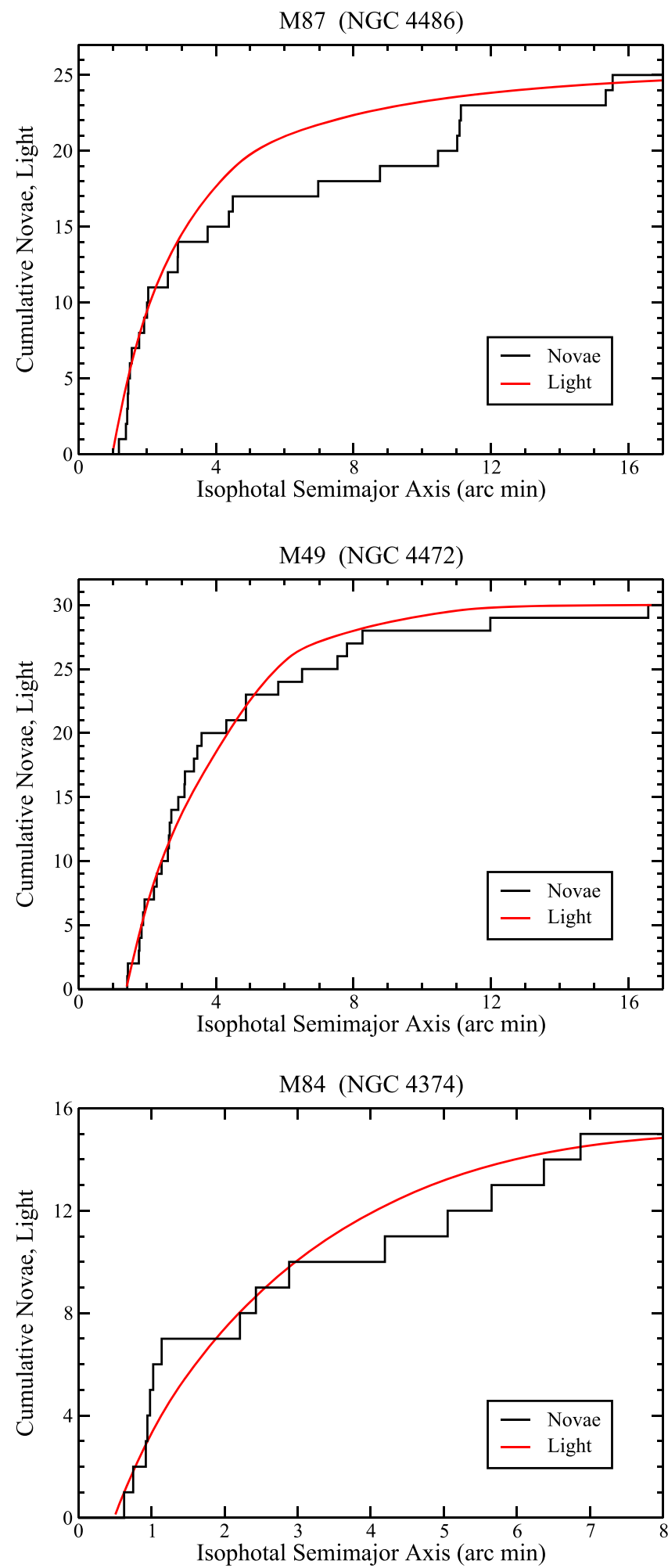

Figure 2. Cumulative distributions of the novae detected in each galaxy in our survey plotted against the cumulative distribution of the background light. The inner value of $a$ was set to assure the sample was was complete to the level of the faintest nova. The adopted inner radii of $a=1^{\prime} \cdot 0, a=1{ }^{\prime} \cdot 4$, and $a=0{ }^{\prime} 5$, for M87, M49, and M84, resulted in the omission of 2, 7, and 4 novae from the full nova samples, respectively. KS statistics for the three fits indicate that the novae follow the background light in M87, M49, and M84 with 33\%, 66\%, and $56 \%$ probability.

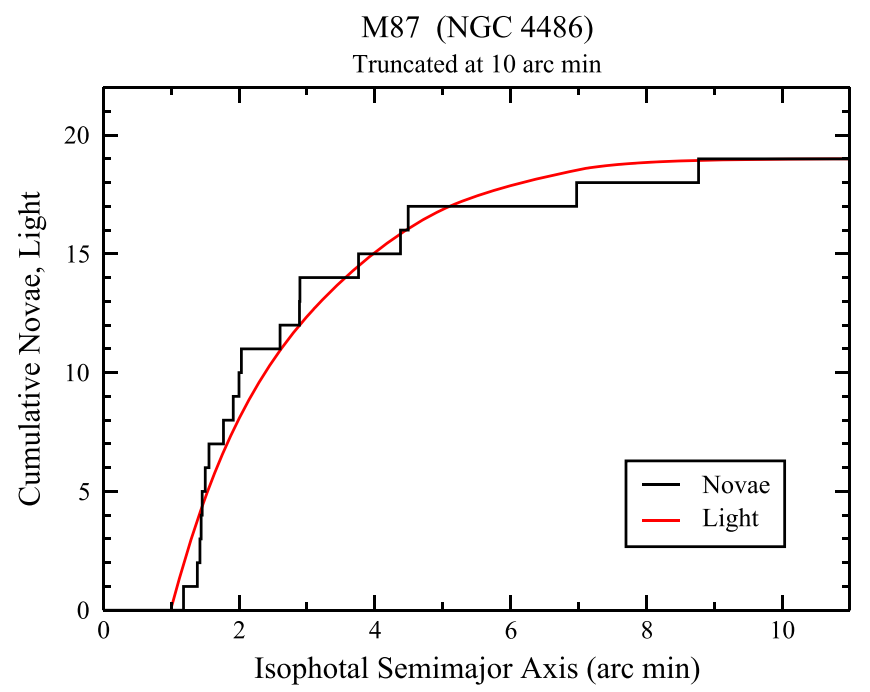

Figure 3. Cumulative distributions of the novae detected in our survey of M87 plotted against the cumulative distribution of the background light truncated to $10^{\prime}$. A KS test shows that the distributions match with $81 \%$ probability.

\section{RESULTS AND DISCUSSION}

\subsection{Relative Nova Rates}

As described earlier, the homogeneous nature of our survey, where we monitored each galaxy in a consistent fashion, allows us to estimate the relative nova rates simply by comparing the relative numbers of novae detected. We have found that the relative number of novae discovered in each galaxy (27 in M87, 37 in M49, and 19 in M84) is in good agreement with that predicted under the null hypothesis that the nova rate scales directly with galaxy luminosity. This is true whether we consider the entire population of novae discovered in the extended field surrounding each galaxy, or, in the case of M87, whether we consider the more restrictive sample (21 novae) based on the best match of the cumulative nova distribution with the background light. Thus, based solely on the relative numbers of novae discovered in the three galaxies, we find no evidence that the GC specific frequency plays a significant role in the nova production in the host galaxies.

\subsection{Absolute Nova Rates}

Although our program was designed to compare relative nova rates in the three Virgo ellipticals, it is also possible to use our data to estimate absolute nova rates in the galaxies as well. Our procedure follows that employed in earlier studies where we used a Monte Carlo approach to estimate the nova rates (e.g., Franck et al. 2012). In this approach, for a given assumed intrinsic nova rate, $R$, we compute the number of novae that we can expect to observe, $N_{\text {obs }}(R)$, given assumed properties of the nova population (peak brightnesses, and fade rates), our observing cadence, the distance to the galaxy in question and the limiting magnitude of our survey at each epoch, $i$, as a function of position in the galaxy. For a range of plausible values of $R$, the simulation constructs a set of model $\mathrm{H} \alpha$ light curves by randomly selecting peak magnitudes and decay rates 
from a sample of actual $\mathrm{H} \alpha$ nova light curves from M31 (Shafter \& Irby 2001) and M81 (Neill \& Shara 2004). Given the dates of our observations (see Table 2) and the distance modulus to the galaxy (see Table 1), an observed nova luminosity function, $n_{i}(m, R)$, for each epoch is calculated. Following Franck et al. (2012), the number of novae we can expect to observe as predicted by our Monte Carlo analysis is then given by

$$
N_{\mathrm{MC}}(R)=\sum_{i} \sum_{m} C\left(m+\Delta m_{i}\right) n_{i}(m, R),
$$

where $C(m)$ is the completeness as a function of apparent magnitude, which accounts for the variation in limiting magnitude across the galaxy.

The completeness function is estimated through artificial star simulations using the IRAF task ADDSTAR. Artificial novae are generated in several magnitude bins spanning the range of nova magnitudes observed. For each bin, the artificial novae are distributed throughout the galaxy image, with a surface density that follows the background light. Then, employing the same search technique used to discover the real novae, we determine the fraction of artificial novae that we can recover as a function of magnitude. Completeness functions for the three galaxies are shown in Figure 4. Variation in the completeness at different epochs, which may differ slightly in exposure time and seeing, is compensated for by estimating an offset, $\Delta m_{i}=m_{\mathrm{lim}, 0}-m_{\mathrm{lim}, i}$, between the epoch used in the artificial star tests and the other epochs.

The most probable nova rate for a given galaxy is given when the number of novae actually observed, $N_{\text {obs }}$, matches the number of novae predicted by our Monte Carlo analysis, $N_{\mathrm{MC}}(R)$. The Monte Carlo program repeats this procedure 100,000 times and records the number of times $N_{\mathrm{MC}}(R)=N_{\text {obs }}$ for each galaxy. The number of matches as a function of $R$, normalized to the total number of trials, produces the probability distribution of nova rates for each galaxy shown in Figure 5. The peak of this distribution represents the most likely intrinsic nova rate, $R$, with $1 \sigma$ errors estimated by assuming Bi-Gaussian fits to the probability distributions (Buys $\&$ De Clerk 1972). We have also computed a nova rate for M87 based upon the truncated sample with $a<10^{\prime}$. A summary of the nova rates for each galaxy is presented in Table 6 . We note that the rates determined for M87 and M49, in particular, are somewhat higher that those found previously in studies by Shafter et al. (2000) and Ferrarese et al. (2003). Despite our higher nova rate for $\mathrm{M} 87$, our value of $154_{-19}^{+23} \mathrm{yr}^{-1}$ for the full sample falls short of the rate of $\sim 200-300 \mathrm{yr}^{-1}$ suggested by Shara \& Zurek (2002) from early HST data, but is similar to that found by Mizusawa et al. (2013).

In addition to computing nova rates, we have also determined the $K$-band luminosity-specific nova rate, $\nu_{K}$ for each galaxy from the data given in Table 1 . In all cases we find values of $\nu_{K}$ of $\sim 3$ novae per year per $10^{10}$ solar luminosities in the $K$ band. Our results are somewhat higher, but still marginally consistent with the findings of Williams \& Shafter (2004) and Güth et al. (2010) who found for a large sample of galaxies that $\nu_{K} \approx 2 \pm 1$ independent of Hubble type. Our $K$ band luminosity-specific nova rates are presented in Table 6.

\section{GC NOVAE}

Two of the novae discovered in our survey, one in M87 and one in M84, appear to be spatially coincident with GCs
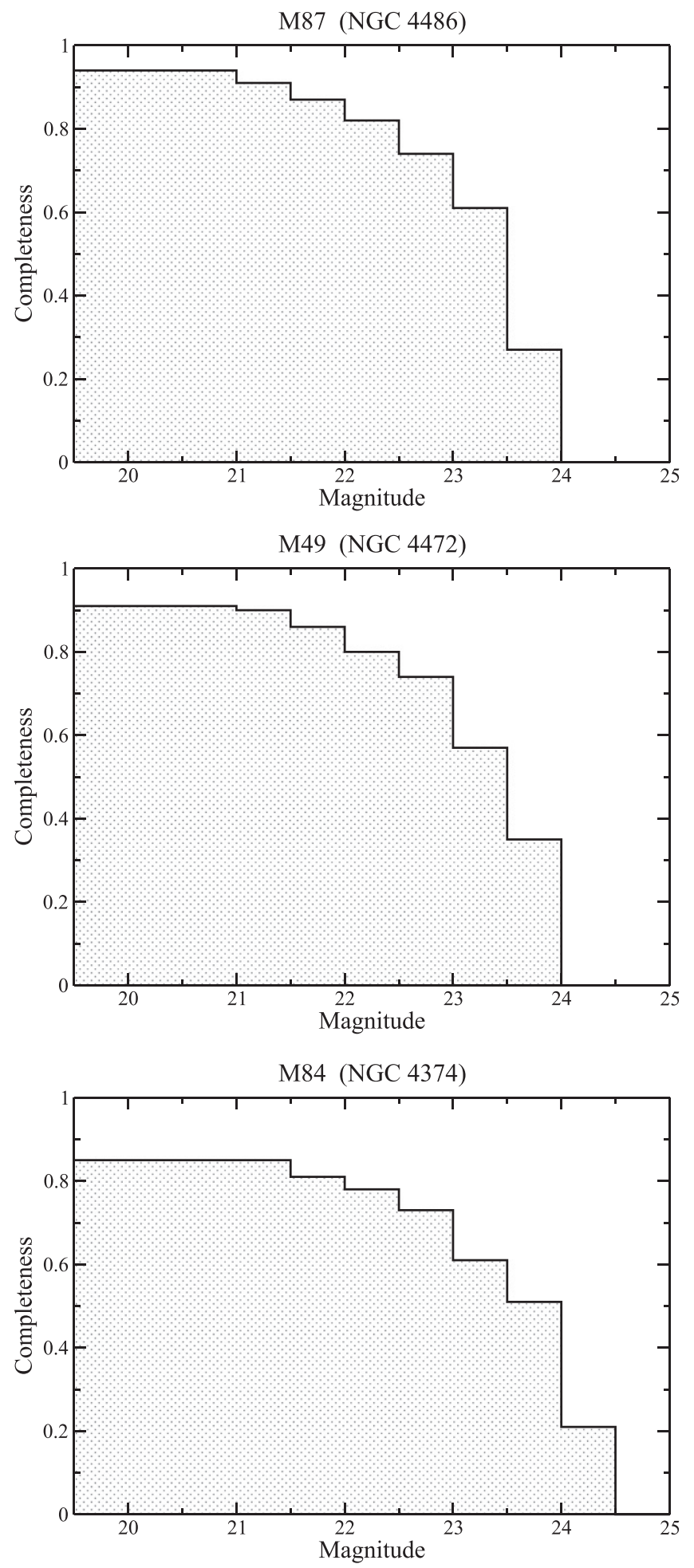

Figure 4. Completeness functions, $C(m)$, showing the fraction of artificial novae recovered in our artificial nova simulations for the different galaxies as a function of apparent magnitude.

associated with these galaxies. No GC novae were identified in M49. The GC nova candidates were discovered from the detection of residual light in differenced images at the positions of known GCs. Instrumental magnitudes were measured for the globulars in question, as well as for several nearby clusters, for all four epochs. While the magnitudes of the surrounding clusters remained reasonably constant, the two GCs thought to 

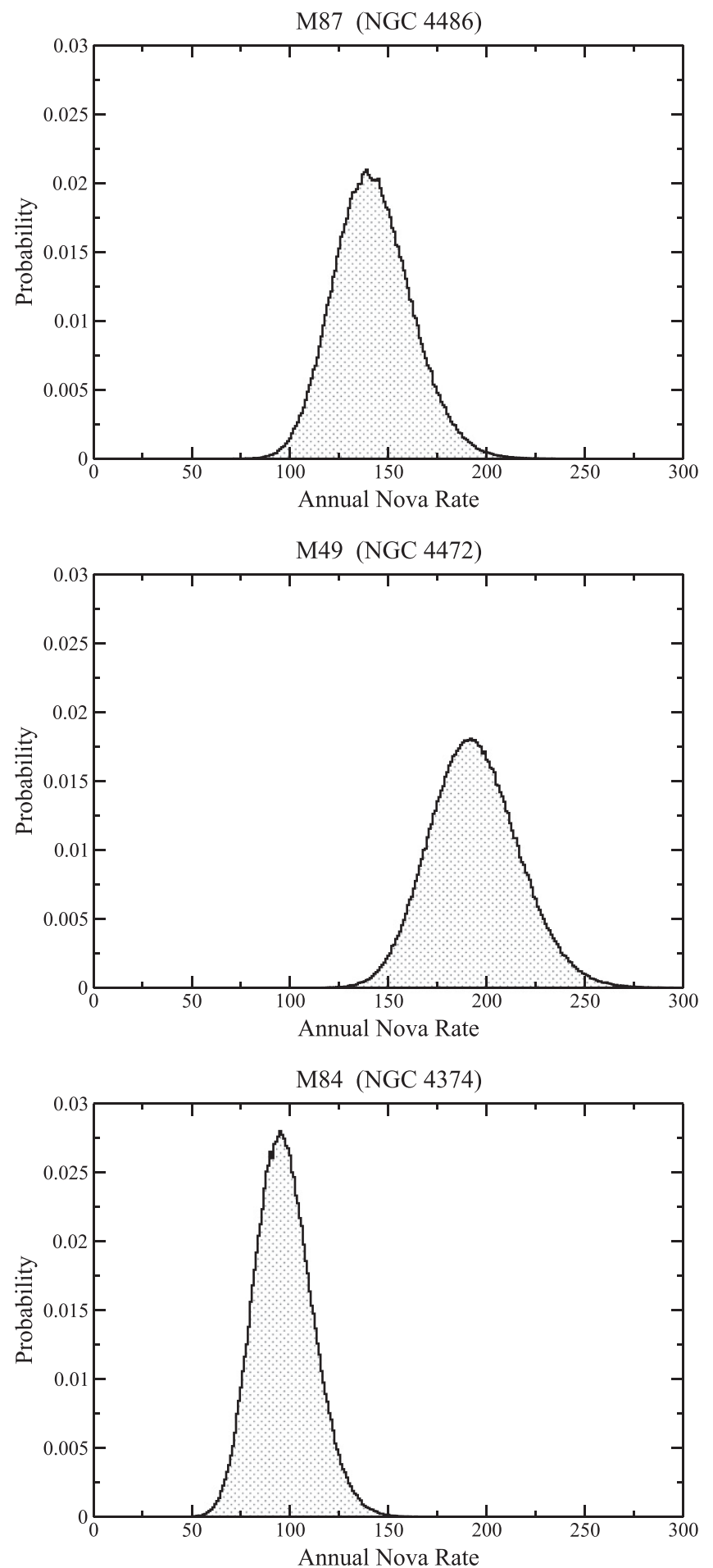

Figure 5. Probability distributions of the annual nova rates, $R$. The peak of each distribution represents the most likely nova rate, with $1 \sigma$ errors determined assuming that the distribution is Bi-Gaussian (see text).

host novae were seen to increase significantly in brightness in only one epoch. Figure 6 shows images of the M84 and M87 GC nova fields from epochs when the novae were detected, quiescent epochs, and their differences. We attribute the residual light to erupting novae in these clusters. Nova magnitudes were estimated by subtracting the contribution of the host GC determined from quiescent epochs. The resulting nova magnitudes given in Tables 3 and 5, were consistent with
Table 6

Nova Rates

\begin{tabular}{lccc}
\hline \hline Galaxy & $N_{\text {obs }}$ & $R\left(\mathrm{yr}^{-1}\right)$ & $\nu_{K}$ \\
\hline M87 & 27 & $154_{-19}^{+23}$ & $\left(10^{10} L_{\odot, K} \mathrm{yr}\right)^{-1}$ \\
M87 $_{T}^{\mathrm{a}}$ & 21 & $124_{-16}^{+23}$ & $3.8 \pm 1.0$ \\
M49 & 37 & $189_{-22}^{+26}$ & $3.1 \pm 0.8$ \\
M84 & 19 & $95_{-14}^{+15}$ & $3.4 \pm 0.6$ \\
\hline
\end{tabular}

Note.

a Only novae interior to an isophotal semimajor axis of $10^{\prime}$ have been considered in this analysis.
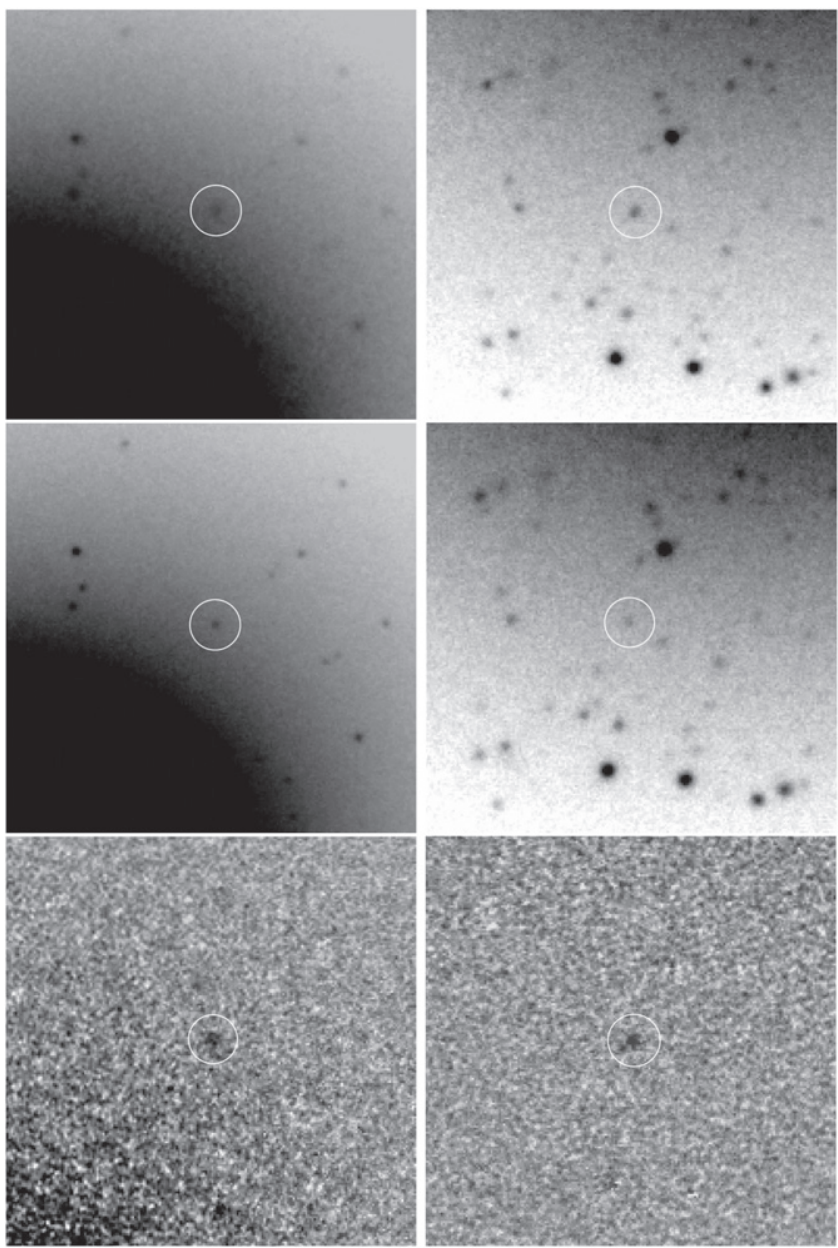

Figure 6. Left column: images of the M84 GC CLW2011-033 from Epoch 2 (with nova, top), from Epoch 4 (without nova), and their difference. Right column: images of the M87 GC PJB2009-1667 from Epoch 4 (with nova, top), from Epoch 1 (without nova), and their difference. In both cases residual light in the GCs is detected between epochs that we attribute to erupting novae. north is up and east to the left, with a scale of $\sim 40^{\prime \prime}$ on a side.

those of the field novae, providing further evidence that the cluster brightenings were indeed the result of novae erupting from within the cluster.

The PSFs of the GCs with erupting novae were consistent with those for other GCs, and were indicative of a single, unresolved light source. The centroid of the PSF for the GCs with erupting novae were coincident with their quiescent counterparts in all epochs to within 0 ". 1 , much less than the 
astrometric error of $1^{\prime \prime}$. We are sufficiently confident that these events are in fact GC novae to include them in our final count.

The M84 GC, CLW2011:033, is a relatively blue cluster characterized by $g=22.853 \pm 0.016$ and $g-z=0.997 \pm$ 0.024 (Chies-Santos et al. 2011). The cluster appears to be metal-poor and relatively massive, being $\sim 1$ mag brighter than the turnover magnitude. On the other hand the M87 cluster, PJB2009:1667, is characterized by $V=23.235 \pm 0.007$ and $V-I=1.183 \pm 0.010$ (Peng et al. 2009). This cluster is redder than the M84 cluster, more metal-rich, with a flux that is $\sim 0.5$ mag brighter than the turnover magnitude.

Despite predictions that novae may be common in GCs, relatively few have been reported. There have been just seven novae identified with GCs: four in M31 (M31N 2007-06b in Bol 111 Shafter \& Quimby 2007, CXO J004345 in Bol 194 Henze et al. 2009, M31N 2010-10f in Bol 126 Cao et al. 2012; Henze et al. 2013, and possibly an anonymous source in Bol 383 Peacock et al. 2010a), two in the Galaxy (T Sco (Nova Sco 1860) in M80 Luther 1860; Pogson 1860, and possibly Nova Oph 1938 in M14 Hogg 1964; Margon et al. 1991), and an anonymous source in an M87 cluster (Shara et al. 2004). Until now, the latter object was the only GC nova that has been reported in M87, with no cluster novae known in either M49 or M84. Clearly, the paucity of novae detected in GCs to date is in part a consequence of the difficulty in discovering novae against the bright background light of the cluster.

The discovery of two GC novae from our survey provides clear evidence that GCs do contribute some fraction of a galaxy's nova system population, and that novae seem to be enhanced in GCs relative to the field. We can estimate the GC enhancement by estimating the mass in GCs in the three galaxies and comparing it with the integrated galaxy masses. The total number of GCs is estimated to be 13,200 in M87 (Harris et al. 1998), 5900 in M49 (Rhode \& Zepf 2004), and 1775 in M84 (Gomez \& Richtler 2004). Using a mean GC mass of $2.4 \times 10^{5} M_{\odot}$ appropriate for massive Virgo ellipticals (McLaughlin 1999), we estimate that the GC systems have a combined mass of $\sim 5 \times 10^{9} M_{\odot}$. For the galaxy masses, we adopt $7.6 \times 10^{11} M_{\odot}, 8.1 \times 10^{11} M_{\odot}$, and $4.0 \times 10^{11} M_{\odot}$ for M87, M49, and M84, respectively (Angeletti \& Giannone 1997, Table 1), yielding a combined mass of $\sim 2.0 \times 10^{12} M_{\odot}$. Thus, GCs represent $\sim 2.5 \times 10^{-3}$ of the total mass in the three galaxies. Given that we have detected two GC novae out of a total of 83 novae discovered in our survey, we estimate that novae erupt $\sim 10$ times more frequently in GCs than they do in the field.

Shara et al. (2004) estimated that novae occur in M87 GCs with a frequency, $f \sim 4 \times 10^{-3}$ novae per GC per year, which is $\sim 2$ orders of magnitude higher than what would be expected if novae were not enhanced relative to the field. In our observations of M87 we have discovered one GC nova out of 27 total novae. Assuming M87 has 13,200 GCs and an overall nova rate of $\sim 150 \mathrm{yr}^{-1}$, we estimate a GC rate of $f \sim 4 \times 10^{-4}$ novae per cluster per year, or about an order of magnitude less than that estimated by Shara et al. (2004) for this galaxy.

We can estimate the significance of the GC enhancement as follows. Let $p=2.5 \times 10^{-3}$ be the fraction of a galaxy's mass in GCs. The probability of observing $N$ or more GC novae out of a sample of $M$ total novae assuming no $\mathrm{GC}$ enhancement is simply

$$
P_{\geqslant N, M}=\sum_{N=2}^{\infty} \frac{M !}{N !(M-N) !} p^{N}(1-p)^{M-N} .
$$

The probability of observing 2 or more GC novae out of 83 novae is then $P_{\geqslant 2,83} \simeq 0.018$, or $\sim 1.8 \%$. In other words, we conclude that novae are enhanced in GCs with $98.2 \%$ confidence. Our estimate likely represents a lower limit to the true GC nova enhancement given the difficulty in identifying novae against the GC background light. The selection effect is amplified when we consider that the most luminous GC have the densest cores, and are thus the most likely to form nova progenitor binaries.

Novae discovered in GCs can provide insights into the dynamics of the clusters and isolate the effects of physical parameters such as age and metallicity in the (primarily) simple stellar populations of GCs. Low mass X-ray binaries, which are the only other binary systems that can be studied in Virgo cluster galaxies, provide an interesting point of reference. These compact neutron star and white dwarf systems are dynamically enhanced in GCs (Clark 1975; Hills 1976) by a factor of several hundred, and show several clear trends. The $\mathrm{X}$-ray binary rate is correlated with the masses and metallicities of the host GCs. X-ray binaries are preferentially found the most massive GCs because the density of GCs increases with mass leading to a very strong correlation between the dynamical interaction rate, cluster luminosity and X-ray binary rate (Jordán et al. 2007; Peacock et al. 2010b). The fact that the two GCs that host novae in our survey are $~ 0.5-1$ mag brighter than the turnover magnitude is consistent with this mass trend. We note, however, that because the peak luminosity of novae rival those of GCs it is very likely that some candidates in the most luminous GCs may be missed thereby underestimating the dynamical formation rate and the effect of GC mass (and the closely connected dynamical interaction rate) on nova formation.

Extragalactic studies of X-ray binaries in GCs have firmly established that they are three times more abundant in the metal-rich population as compared to the metal-poor one in the typical bimodal GC populations in galaxies, a surprising effect that was hinted at in early Milky Way and M31 observations (Silk \& Arons 1975; Bellazzini et al. 1995; Kundu et al. 2007). The M84 GC nova found here is metal-poor while the M87 candidate is metal-rich. Interestingly, based on the published metallicities or colors, of the seven other likely or plausible nova GCs only two are likely to be metal-rich. This may hint at the possibility that novae in GCs are not consistent with the metallicity effect seen in X-ray binaries. Clearly no strong conclusions can be drawn form this heterogeneous set of observations with unknown biases. Future systematic observations such as this study are needed to test the intriguing possibility that more massive neutron star and black hole binaries respond differently with metallicity than WD binaries.

\section{CONCLUSIONS}

We have reported the results of a Virgo cluster nova survey to test for a possible relationship between a galaxy's nova rate and its GC specific frequency. In our CFHT survey spanning a total of 4 epochs over two years, we have discovered a total of 
27, 37, and 19 novae in the fields of M87, M49, and M84, respectively. These data have led to nova rate estimates of $154_{-19}^{+23}, 189_{-22}^{+26}$, and $95_{-14}^{+15}$ per year, for the three galaxies. After considering the $K$-band luminosities of the galaxies, we find similar luminosity-specific nova rates, $\nu_{K}$, for M87, M49, and M84 of $3.8 \pm 1.0,3.4 \pm 0.6$, and $3.0 \pm 0.6$ novae per year per $10^{10} L_{\odot, K}$. These values are at the high end of the range of $1-3$ novae per year per $10^{10} L_{K, \odot}$ found by Güth et al. (2010).

The spatial distribution of the M87 novae shows some evidence that the nova population could perhaps be separated into two distinct groups: that of an inner group which follows the background light out to a distance of about $10^{\prime}$, similar to what is seen in M49 and M84, and an outer group of 6 novae clumped between $\sim 14^{\prime}$ and $\sim 16^{\prime}$. As mentioned previously, M87 appears to have an extended halo of diffuse light that is populated with lower luminosity elliptical galaxies such as NGC 4478. Thus it is possible that some or all of the novae seen in the outskirts of M87 may not in fact be associated with M87 itself. If we restrict our analysis of M87 to the 21 novae with isophotal radii less than $10^{\prime}$, we find that the cumulative distribution of novae closely follows the background light distribution. For this truncated sample, we find a somewhat smaller nova rate of $124_{-17}^{+23}$ per year for M87, corresponding to a luminosity-specific nova rate of $\nu_{K}=3.1 \pm 0.8$, which is more in line with the values found for other galaxies.

We have discovered two novae spatially coincident with known GCs: one in M87 and one in M84. During the course of our survey a total of 83 novae were discovered in M87, M49, and M84, suggesting that GC novae could comprise $\sim 2.4 \%$ of the novae seen to erupt in these galaxies. This estimate is likely a lower limit given the strong observational selection against the discovery of novae in the brightest clusters (where they may be most likely to occur). Taken together, the GC systems comprise only $\sim 0.23 \%$ of the combined masses of the three galaxies. Thus, taken at face value, it appears that novae may be enhanced by at least an order of magnitude in GC environments. Additional monitoring of the GC systems of Virgo ellipticals, in particular M87, will be required before any definitive conclusions can be reached.

In summary, the results of our survey are consistent with the hypothesis that the nova rates in the three Virgo galaxies studied here are to first order simply proportional to their mass in stars. Despite the detection of two novae associated with GCs in M87 and M84 suggesting that novae may be enhanced in GCs over the field, there is no compelling evidence that the overall nova rates in the Virgo ellipticals are particularly sensitive to the their GC specific frequencies, which vary significantly between the three galaxies.

We thank an anonymous referee for constructive comments on our original manuscript. This work is based on observations obtained with MegaPrime/MegaCam, a joint project of CFHT and CEA/DAPNIA, at the Canada-France-Hawaii Telescope (CFHT) which is operated by the National Research Council (NRC) of Canada, the Institut National des Science de l'Univers of the Centre National de la Recherche Scientifique (CNRS) of France, and the University of Hawaii. A.W.S. and C.C. acknowledge financial support through NSF grant AST1009566. C.J.P. acknowledges financial support from the Natural Sciences and Engineering Research Council of Canada.

\section{REFERENCES}

Alard, C., \& Lupton, R. H. 1998, ApJ, 503, 325

Angeletti, L., \& Giannone, P. 1997, A\&A, 321, 343

Bellazzini, M., Pasquali, A., Federici, L., Ferraro, F. R., \& Pecci, F. F. 1995 , ApJ, 439, 687

Boulade, O., Charlot, X., Abbon, P., et al. 2003, Proc. SPIE, 4841, 72

Brodie, J. P., \& Strader, J. 2006, ARA\&A, 44, 193

Buys, T. S., \& De Clerk, K. 1972, AnaCh, 44, 1273

Cao, Y., Kasliwal, M., \& Neill, J. D. 2012, ApJ, 752, 133

Capaccioli, M., della Valle, M., Rosino, L., \& D’Onofrio, M. 1989, AJ, 97, 1622

Chies-Santos, A. L., Larsen, S. S., Wehner, E. M., et al. 2011, A\&A, 525, 19

Ciardullo, R., Ford, H. C., Neill, J. D., Jacoby, G. H., \& Shafter, A. W. 1987, ApJ, 318, 520

Ciardullo, R., Shafter, A. W., Ford, H. C., et al. 1990a, ApJ, 356, 472

Ciardullo, R., Tamblyn, P., Jacoby, G. H., Ford, H. C., \& Williams, R. E. 1990b, AJ, 99, 1079

Clark, G. W. 1975, ApJ, 199, 143

Coelho, E. A., Shafter, A. W., \& Misselt, K. A. 2008, ApJ, 686, 1261

Cohen, J. G. 1986, AJ, 92, 1039

Crampton, D., Hutchings, J. B., Cowley, A. P., Schade, D. J., \& van Speybroeck, L. P. 1984, ApJ, 284, 663

Curtin, C., Shafter, A. W., \& Pritchet, C. 2014, BAAS, 223, 113.03

Darnley, M. J., Bode, M. F., Kerins, E., et al. 2004, MNRAS, 353, 571

Darnley, M. J., Bode, M. F., Kerins, E., et al. 2006, MNRAS, 369, 257

Darnley, M. J., et al. 2014, A\&A, 563, 9

de Vaucouleurs, G. 1948, AnAp, 11, 247

de Vaucouleurs, G., de Vaucouleurs, A., Corwin, H. G., Jr., et al. 1991, Third Reference Catalogue of Bright Galaxies (New York: Springer)

della Valle, M., Rosino, L., Bianchini, A., \& Livio, M. 1994, A\&A, 287, 403

Di Stefano, R., et al. 2002, ApJ, 570, 618

Edmonds, P. D., Gilliland, R. L., Heinke, C. O., \& Grindlay, J. E. 2003, ApJ, 596, 1197

Ferrarese, L., Côté, P., \& Jordán, A. 2003, ApJ, 599, 1302

Franck, J. R., Shafter, A. W., Hornoch, K., \& Misselt, K. A. 2012, ApJ, 760, 13

Frogel, J. A., Persson, S. E., Matthews, K., \& Aaronson, M. 1978, ApJ, 220, 75

Gomez, M., \& Richtler, T. 2004, A\&A, 415, 499

Grindlay, J. E. 1988, IAUS, 126, 347

Güth, T., Shafter, A. W., \& Misselt, K. A. 2010, ApJ, 720, 1155

Harris, W. E., Harris, G. L. H., \& McLaughlin, D. E. 1998, AJ, 115, 1801

Heinke, C. O., Grindlay, J. E., Lugger, P. M., et al. 2003, ApJ, 598, 501

Henze, M., Pietsch, W., Haberl, F., et al. 2009, A\&A, 500, 769

Henze, M., Pietsch, W., Haberl, F., et al. 2013, A\&A, 549, 120

Henze, M., et al. 2014, A\&A, 563, 8

Hills, J. G. 1976, MNRAS, 175, 1

Hogg, D. E. 1964, JRASC, 58, 203

Iben, I., Jr., Fujimoto, M. Y., \& MacDonald, J. 1992, ApJ, 384, 580

Jordán, A., McLaughlin, D. E., Côté, P., et al. 2007, ApJS, 171, 101

Katz, J. I. 1975, Natur, 253, 698

Knigge, C., Zurek, D. R., Shara, M. M., \& Long, K. S. 2002, ApJ, 579, 752

Kolb, U. 1995, in Cataclysmic Variables, ed. A. Bianchini, M. della Valle, \& M. Orio (Astrophysics and Space Science Library, Vol. 205; Dordrecht: Kluwer), 511

Kormendy, J., Fisher, D. B., Cornell, M. E., \& Bender, R. 2009, ApJS, 182,216

Kundu, A., Maccarone, T. J., \& Zepf, S. E. 2007, ApJL, 574, L5

Livio, M. 1992, ApJ, 393, 516

Luther, E. 1860, AN, 53, 293

Madrid, J. P., Sparks, W. B., Ferguson, H. C., Livio, M., \& Macchetto, D. 2007, ApJL, 654, L41

Margon, B., Anderson, S. F., Downes, R. A., Bohlin, R. C., \& Jakobsen, P. 1991, ApJL, 369, L71

McLaughlin, D. E. 1999, AJ, 117, 2398

Michard, R. 1985, A\&AS, 59, 205

Mizusawa, T., Shara, M., \& Zurek, D. 2013, BAAS, 221, 148.07

Monet, D. G., Levine, S. E., Canzian, B., Ables, H. D., Bird, A. R., et al. 2003, AJ, 125, 984

Neill, J. D., \& Shara, M. M. 2004, AJ, 127, 816

Neill, J. D., Shara, M. M., \& Oegerle, W. R. 2005, ApJ, 618, 692

Peacock, M. B., Maccarone, T. J., Knigge, C., et al. 2010a, MNRAS, 402, 803

Peacock, M. B., Maccarone, T. J., Kundu, A., \& Zepf, S. E. 2010b, MNRAS, 407, 2611

Peng, E. W., Jordán, A., Blakeslee, J. P., et al. 2009, ApJ, 703, 42

Pietsch, W., Haberl, F., Sala, G., et al. 2007, A\&A, 465, 375 
Pogson, N. 1860, MNRAS, 21, 32

Pooley, D., \& Hut, P. 2006, ApJL, 646, L143

Pooley, D., Lewin, W. H. G., Homer, L., et al. 2002, ApJ, 569, 405

Ritter, H., Politano, M., Livio, M., \& Webbink, R. F. 1991, ApJ, 376, 177

Rhode, K. L., \& Zepf, S. E. 2004, AJ, 127, 302

Shafter, A. W. 2008, in Classical Novae, ed. M. Bode \& A. Evans (2nd ed.; Cambridge: Cambridge Univ. Press), 335

Shafter, A. W., Ciardullo, R., \& Pritchet, C. J. 2000, ApJ, 530, 193

Shafter, A. W., \& Irby, B. K. 2001, ApJ, 563, 749

Shafter, A. W., Pritchet, C., Neill, J. D., et al. 2013, BAAS, 221, 148.06

Shafter, A. W., \& Quimby, R. M. 2007, ApJL, 671, L121

Shara, M. M. 2006, AJ, 131, 2980
Shara, M. M., \& Zurek, D. R. 2002, in AIP Conf. Proc. 637, Classical Nova Explosions (Melville, NY: AIP), 457

Shara, M. M., Zurek, D. R., Baltz, E. A., Lauer, T. R., \& Silk, J. 2004, ApJL, 605, L117

Silk, J., \& Arons, J. 1975, ApJL, 200, L131

Tonry, J. L., Dressler, A., Blakeslee, J. P., et al. 2001, ApJ, 546, 681

Townsley, D. M., \& Bildsten, L. 2005, ApJ, 628, 395

Tutukov, A. V., \& Yungelson, L. R. 1995, in Cataclysmic Variables, ed. A. Bianchini, M. della Valle \& M. Orio (Astrophysics and Space Science Library, Vol. 205; Dordrecht: Kluwer), 495

Williams, S. J., \& Shafter, A. W. 2004, ApJ, 612, 867

Yungelson, L., Livio, M., \& Tutukov, A. 1997, ApJ, 481, 127 